\section{A simplified immunohistochemical classification of skeletal muscle fibres in mouse}

\author{
M. Kammoun, 1,2 I. Cassar-Malek, ${ }^{1,2}$ \\ B. Meunier, ${ }^{1,2}$ B. Picard ${ }^{1,2}$ \\ ${ }^{1}$ INRA, UMR 1213 Herbivores, Saint- \\ Genès-Champanelle, France \\ ${ }^{2}$ Clermont University, VetAgro Sup, UMR \\ 1213 Herbivores, Clermont-Ferrand, \\ France
}

\section{Abstract}

The classification of muscle fibres is of particular interest for the study of the skeletal muscle properties in a wide range of scientific fields, especially animal phenotyping. It is therefore important to define a reliable method for classifying fibre types. The aim of this study was to establish a simplified method for the immunohistochemical classification of fibres in mouse. To carry it out, we first tested a combination of several anti myosin heavy chain (MyHC) antibodies in order to choose a minimum number of antibodies to implement a semi-automatic classification. Then, we compared the classification of fibres to the MyHC electrophoretic pattern on the same samples. Only two anti MyHC antibodies on serial sections with the fluorescent labeling of the Laminin were necessary to classify properly fibre types in Tibialis Anterior and Soleus mouse muscles in normal physiological conditions. This classification was virtually identical to the classification realized by the electrophoretic separation of MyHC. This immunohistochemical classification can be applied to the total area of Tibialis Anterior and Soleus mouse muscles. Thus, we provide here a useful, simple and time-efficient method for immunohistochemical classification of fibres, applicable for research in mouse.

\section{Introduction}

Skeletal muscle fibres are classified accor ding to their contractile and metabolic properties. The contractile properties depend on the composition in myosin heavy chain isoform (MyHC) (for a review see Schiaffino and Reggiani). ${ }^{1}$ MyHC encoded by a multigene family $^{2}$ is the major component of the contractile apparatus combining with actin to form the actomyosin complex which is responsible for the elastic and contractile properties of muscle. Evolution in the knowledge about MyHC isoforms led to progression in the classification of fibres using different techniques. ${ }^{3}$ Firstly, the histochemical technique was based on the revelation of the ATPase activity of MyHC and allowed to distinguish between slow and fast muscle fibres. The myosin ATPase activity is higher in fast than in slow muscles and correlates with muscle shortening speed. ${ }^{4}$ Then, the combination of different $\mathrm{pH}$ of preincubation for the revelation of ATPase activity revealed three types of fibres mainly I, IIA and IIB. ${ }^{5,6}$ The discovery of the activity of oxidative enzymes (e.g., the Succinate dehydrogenase, SDH) helped distinguish oxidative and non oxidative fibres. ${ }^{7} \mathrm{~A}$ combination of methods to simultaneously detect contractile and metabolic properties can detect slowoxidative fibres, fast glycolytic and fast oxidative fibres. $^{3}$ Then, with the progress of immunology, anti MyHC monoclonal antibodies were produced. Their use by immunohistochemistry on serial sections enabled the detection of four types of fibres in rat, mouse, rabbit, pig muscles: I, IIA, IIX (or IID) and IIB. ${ }^{8}$ The development of electrophoretic separation of MyHC according to their molecular weights also revealed the existence of four MyHC in adult rodent muscles. ${ }^{9}$ Moreover, the use of monoclonal antibodies demonstrated that some fibres called hybrid fibres contain several isoforms of MyHC. In situ hybridization analysis on single fibre, confirmed that rodent muscles contain a spectrum of fibre types, including hybrid fibres with preferential combinations of MYH transcripts, according to the following sequence: I $\leftrightarrows$ I / IIA $\leftrightarrows$ IIA $\leftrightarrows$ IIA IIX $\leftrightarrows$ IIX $\leftrightarrows$ IIX/ IIB $\leftrightarrows$ IIB. $^{10}$

Among the different techniques, immunohistochemistry is the most accurate as it makes it possible to distinguish pure and hybrid fibres. This method has been used for the analysis of skeletal muscle in different species; ${ }^{3,11,12}$ for the studies in mice, different antibodies are available. ${ }^{13,14}$ Several hundred of fibres might reasonably be analyzed per biological sample by comparing serial sections using different anti MyHC antibodies. The manual analysis of the different sections is laborious and time consuming, that is why several authors developed semi-automatic image analysis softwares. ${ }^{15,16}$

The aim of the present study was to adapt the method of Meunier et al. for the classification of contractile fibre types in mouse. ${ }^{16}$ Our objective was to use a minimum number of antibodies to reduce the number of serial sections to be compared. We first tested a combination of several anti MyHC antibodies. Then, we validated the classification of the fibres obtained by immunohistochemistry through a comparison with the MyHC electrophoretic pattern on the same samples.
Correspondence: Brigitte Picard, INRA, UMR 1213 Herbivores, F-63122 Saint-GenèsChampanelle, France.

Tel. +33.4.73624056 - Fax: +33.4.73624639. E-mail: brigitte.picard@clermont.inra.fr

Keywords: skeletal muscle, mouse, myosin heavy chain, immunohistochemistry, electrophoresis, image analysis.

Contributions: MK, analytical immunohistochemistry experiments, data interpretation, manuscript drafting; ICM, experiment co-manager, manuscript drafting; BM, image analysis, manuscript drafting; BP, experiment co-manager, myosin and immunohistochemistry studies specialist, manuscript drafting.

Conflict of interests: the authors declare no conflict of interests.

Acknowledgments: we are grateful to the experimental plant for nutrition and microbiology of INRA (Clermont-Ferrand/Theix) for animal management. We also thank Christiane Barboiron for her excellent technical support and advice on immunohistochemistry.

Received for publication: 30 July 2013. Accepted for publication: 14 April 2014.

This work is licensed under a Creative Commons Attribution NonCommercial 3.0 License (CC BYNC 3.0).

(C) Copyright M. Kammoun et al., 2014

Licensee PAGEPress, Italy

European Journal of Histochemistry 2014; $58: 2254$ doi:10.4081/ejh.2014.2254

\section{Materials and Methods}

\section{Animals and experimental procedure}

Two muscles known to have a different composition of fibre types were studied, namely the m. Soleus (SOL) and m. Tibialis Anterior (TA). According to the literature, the SOL is a slow oxidative muscle and the TA a fast glycolytic muscle. ${ }^{17,18}$ Both muscles were dissected from anaesthetized male C57BL6 mice at 12 weeks of age $(n=8)$. Following dissection, they were frozen in liquid nitrogen and stored at $80^{\circ} \mathrm{C}$ for further analysis.

\section{Immunohistochemical detection of myosin heavy chains}

\section{MyHC antibodies}

For contractile fibre type determination, in order to detect slow and fast MyHC isoforms, we chose anti MyHC antibodies according to the data available for mouse skeletal muscle (Table 1). Six antibodies were tested on serial 
sections. BA-D5 specific for MyHC I, SC71 specific for MyHC Ila, BF-F3 specific for MyHC IIb, ${ }^{19}$ S5-8H2 for MyHC I, IIx and IIb. These antibodies were purchased from AGRO-BIO (La Ferté Saint Aubin, France). ${ }^{20}$ N2.261, which reveals MyHC I and IIa, and RTD-9 labelling MyHC II ${ }^{19}$ were purchased from Enzo Life Sciences (ELS) (Lyon, France). The reactivity of these antibodies has been validated on mouse muscles. ${ }^{17}$

\section{Immunohistochemical revelation}

Serial transverse sections (10- $\mu \mathrm{m}$ thick) were obtained from each muscle sample using a cryostat (Cryo-star HM 560, Microm International $\mathrm{GmbH}$, Germany) at $-26^{\circ} \mathrm{C}$, mounted on glass slides and stained using immunohistochemical methods. The sections were blocked to eliminate non specific binding in 5\% BSA diluted in phosphate-buffered saline (PBS) for $10 \mathrm{~min}$. The cross-sections were then incubated with primary antibodies in a humidified chamber for one hour in the dark at $37^{\circ} \mathrm{C}$ (dilution conditions illustrated in Table 2). After washing in phosphate-buffered saline, the sections were then incubated with the secondary antibodies for $30 \mathrm{~min}$ in the dark at $37^{\circ} \mathrm{C} .{ }^{16}$ Each anti MyHC antibody was used double labelling with anti laminin antibody on the same section. The MyHC isoforms were revealed by an Alexa Fluor 488 labeled goat anti-mouse IgG secondary antibody (A11001, Invitrogen, Carlsbad, CA, USA, dilution 1/200). For BF-F3, we used an Alexa Fluor 546 anti-mouse IgM secondary antibody (A11010, Invitrogen ; dilution 1/1000). The cell outline was stained using a rabbit anti-laminin primary polyclonal antibody (L9393 SigmaAldrich, St. Louis, M0, USA; Table 2) and a goat anti-rabbit IgG Cy3-labeled secondary antibody (111-165-008, Jackson Immunoresearch Labs, West Grove, PA, USA, dilution 1/200). After further washing in PBS, sections were mounted with cover slips using Fluoromount (F4680, Sigma-Aldrich). Finally, negative control experiments (section incubated without specific primary antibodies and anti-laminin antibody) were performed with all immunohistochemical staining to demonstrate the non-specific reactivity.

\section{Image acquisition and analysis}

Histological sections were visualized under an Olympus fluorescence microscope BX 51, using a 10X objective $(\mathrm{NA}=0.3)$ and adequate band pass filter (Alexa 488: excitation filter 460-495, emission filter 510-550, dichromatic mirror 505LP; Cy3: excitation filter 530-550, emission filter 575-625, dichromatic mirror 565LP) as described by Meunier et al. ${ }^{16}$ High resolution grayscale images were acquired with an Olympus cooled digital camera DP-72 with cell-F software (Olympus Soft Imaging Solutions, Münster, Germany). For each field

Table 1. Summary of the responses of different anti Myosin Heavy Chains (MyHC) antibodies in mouse Soleus and Tibialis Anterior muscles.

\begin{tabular}{lcccc}
\hline Isoforms & MyHC-I & MyHC-Ila & MyHC-IIx & MyHC-Ilb \\
BA-D5 & + & - & - & - \\
SC-71 & - & + & - & - \\
N2.261 & $+/$ & + & - & - \\
S5-8H2 & + & - & + & + \\
BF-F3 & - & - & - & + \\
RTD-9 & - & - & + & - \\
\hline
\end{tabular}

According to Duris et $a l .{ }^{11}$, and Schiaffino et al ${ }^{27}$; -, unlabeled; +/-, intermediate; +, labeled.

of each serial section, two images corresponding to the laminin and MyHCs labelling respectively were digitized. Image processing and image analysis were realized with the FibTypFluo program developed in visual basic under the Visilog 6.9 Professional Software (Noesis, Gif-sur-Yvette, France).

This program is described in a flow chart ${ }^{16}$ and permits to automatically determine the muscle fibre type proportion (\%), mean crosssectional area (CSA) per type and fibre density (number per $\mathrm{mm}^{2}$ ) for an average 300 fibres per serial image.

\section{Electrophoretic separation of $\mathrm{MyHC}$ isoforms}

Muscle proteins were extracted with a buffer containing $0.5 \mathrm{M} \mathrm{NaCl}, 20 \mathrm{mM} \mathrm{NaPPi}, 50$ mM Tris, 1 mM EDTA and 1 mM DTT according to the protocol described by Picard et al. ${ }^{21}$ Samples were homogenized with a polytron. Following centrifugation at $2500 \mathrm{x}$ g for $10 \mathrm{~min}$ at $4^{\circ} \mathrm{C}$, the supernatants were diluted $1: 1(\mathrm{v} / \mathrm{w})$ with glycerol and stored at $-20^{\circ} \mathrm{C}$. The protein concentration of the samples was determined according to the method of Bradford. ${ }^{22}$

The samples were suspended in 1:1 v/v in a basic $2 \times$ Laemmli solution containing $4 \%$ w/v sds, 10\% v/v $\beta$-mercaptoethanol, 20\% v/v glycerol, $125 \mathrm{mM}$ Tris (pH 6.8) and $0.01 \% \mathrm{w} / \mathrm{v}$ pyronin $\mathrm{Y}$, incubated at room temperature for $10 \mathrm{~min}$ and then heated $\left(70^{\circ} \mathrm{C}\right)$ for $10 \mathrm{~min}$. The MyHC isoforms were separated with SDS glycerol gel electrophoresis on $160 \times 160 \times 1.5 \mathrm{~mm}$ plates using a Hoefer system in accordance with the method of Laemmli. ${ }^{23}$ The stacking gel contained $4 \%$ polyacrylamide with a cross-link of $1.96 \%$. The separating gel was an $8 \%$ polyacrylamide gradient with a cross-link of $1 \%$, as described in Mizunoya et al. ${ }^{24} \mathrm{~A}$ critical factor affecting the resolution of MyHC isoforms was the addition of glycerol to the gel matrix with a final concentration of $35 \%$, according to Sugiura et al. ${ }^{25)}$ Three micrograms of protein were loaded per well onto 0.75 -mm-thick gels. The electrophoresis was run at a constant voltage of $140 \mathrm{~V}$ for $15 \mathrm{~h}$ at $4^{\circ} \mathrm{C}$. After migration, the gels were stained in a solution of
Table 2. Conditions for each primary antibody used in this study.

\begin{tabular}{lc}
\hline Antibody & Dilution \\
Monoclonal anti-bovine BA-D5 & Pure \\
Monoclonal anti-bovine BF-F3 & Pure \\
\hline Monoclonal anti-bovine S5-8H2 & $1 / 2$ \\
Monoclonal anti-bovine SC71 & Pure \\
\hline Monoclonal anti-human N2.261 & $1 / 50$ \\
Monoclonal anti-bovine RTD-9 & Pure \\
\hline Polyclonal anti-human Laminin & $1 / 200$ \\
\hline
\end{tabular}

Coomassie Blue. Gel images were captured using the Expression 10000XL Pro scanner (Epson) and the percentages of abundance of each MyHC (I, IIa, Ilx and IIb) were quantified using ImageQuant TL v7.0 software (GE Healthcare, Fairfield, CT, USA).

\section{Statistical analysis}

Muscle fibre type distribution and cross-sectional areas were expressed as means and standard deviation. Statistical significance of the differences between means for the different combinations of antibodies was assessed by analysis of variance (ANOVA) using the GLM procedure of SAS Software (Statistical Analysis System, Cary, NC, USA). A Student's $t$-test was performed to compare the fibre type distributions obtained with the immunohistochemical method to those obtained with the electrophoresis method. A probability of less than 5\% was considered statistically significant.

\section{Results}

\section{Choice of the anti MyHC antibodies}

Our first aim was to choose the combination of a minimum number of antibodies to obtain a simple and correct typing result while minimizing cost and time. The choice of antibodies was made from the data available in the literature on antibody specificities for mouse skeletal muscles. Among all possible combinations ( 5 antibodies $=23$ combinations of two or more 
antibodies), only those potentially interesting according to the literature were tested according to the muscle studied on one individual.

\section{Soleus muscle}

Several authors demonstrated at protein ${ }^{26}$ or transcript level, ${ }^{17}$ that SOL did not contain IIb MyHC. We therefore used the BF-F3 antibody which specifically recognizes IIB fibres in mouse, ${ }^{27}$ on eight SOL samples and confirmed the absence of MyHC IIb isoform in the SOL (Figure 1). Then, four combinations of antibodies were tested on one mouse and compared to the BA-D5/SC71/S5-8H2 combination used as a reference. As observed in Table 3 , all the combinations revealed equivalent proportions of MyHC in the SOL, with a low coefficient of variation between the different combinations tested. Among the 4 combinations tested, the BA-D5/SC71 was preferred due to the specificity of these antibodies to recognize a single fibre type (Table 1) facilitating image analysis.

\section{Tibialis Anterior muscle}

In the literature, this muscle is said not to contain slow type I fibres. We verified it in the eight samples by using the BA-D5 antibody that is specific for slow MyHC. With this antibody we did not observe any labelling which confirms the absence of type I fibres in the TA (Figure 2). As for the SOL, we tested six different combinations of antibodies (Table 4). All the combinations revealed nearly the same proportions of fibres in the TA. Among the combinations tested, the BF-F3/SC71 combination was chosen because it enables efficient typing of fibres. The pattern of SC71 staining intensities revealed the existence of unlabelled, labelled and intermediary labelled fibres (Figure 2). The intermediary labelled fibres contained less abundance of Ila MyHC than marked fibres, they were IIA-X hybrid fibres. Lastly, no hybrid IIX-B fibres were detected. This was confirmed by using RTD9 as control for MyHC IIx labelling (data not shown).

\section{Characterisation of fibres by image analysis}

Fibre type composition was determined in 8 mice with the two selected combinations of antibodies using semi-automatic image analysis as described by Meunier et al. ${ }^{16}$ The mean percentages of each fibre type were in agreement with the data presented in Tables 3 and 4 , and with data from the literature.

In the $\mathrm{TA}$, we revealed a predominance of type IIB (57\%). A low percentage of hybrid IIA$\mathrm{X}$ fibres (3\%) was observed (Table 5). No hybrid IIX/IIB fibres were observed. This result was confirmed using anti RTD9 antibody (data not shown). The SOL consisted mainly of type IIA and I fibres (respectively $46 \%$ and $43 \%$,
Table 5). We did not observe any hybrid fibres in this muscle. We estimated the total number of fibres on the whole muscle section area in both SOL (muscle area $2 \mathrm{~mm}^{2}$ ) and TA (mus-

cle area $<12 \mathrm{~mm}^{2}$ ) muscles (Figure 3 ). In the TA, 1323 fibres on average (all types combined) were counted i.e., approximately 110 fibres per $\mathrm{mm}^{2}$. The SOL was composed of 724

Table 3. Proportions of I, IIA, IIX, and IIB fibres detected with different combinations of antibodies in the $\mathrm{m}$. Soleus.

\begin{tabular}{lcccc}
\hline Fibres types & $\%$ I & $\%$ IIA & $\%$ IIX & $\%$ IIB \\
BA-D5/N2.261 & 37 & 54 & 9 & 0 \\
BA-D5/S5-8H2 & 40 & 50 & 10 & 0 \\
\hline N2.261/S5-8H2 & 36 & 56 & 8 & 0 \\
BA-D5/SC71 & 38 & 53 & 9 & 0 \\
\hline BA-D5/SC71/S5-8H2 & 40 & 51 & 9 & 0 \\
Mean & 38 & 53 & 9 & 0 \\
\hline CV(\%) & 4 & 4 & 8 & 0
\end{tabular}

$\mathrm{CV}$, coefficient of variation among serial cross sections; the reference combination is in italics.

Table 4. Proportions of I, IIA, IIA-X, IIX, and IIB fibres detected with different combinations of antibodies in the $\mathrm{m}$. Tibialis Anterior.

\begin{tabular}{lccccc} 
Fibres types & \% I & \% IIA & \% IIA-X & \% IIX & \% IIB \\
BF-F3/S5-8H2 & 0 & 4 & 5 & 35 & 56 \\
BF-F3/SC71 & 0 & 5 & 4 & 34 & 57 \\
\hline BF-F3/N2.261 & 0 & 5 & 4 & 33 & 58 \\
S5-8H2/N2.261 & 0 & 6 & 5 & 32 & 56 \\
\hline S5-8H2/SC71 & 0 & 6 & 6 & 32 & 56 \\
BF-F3/SC71/S5-8H2 & 0 & 5 & 4 & 34 & 57 \\
\hline Mean & 0 & 5 & 5 & 33 & 57 \\
CV (\%) & 0 & 19 & 15 & 3 & 2 \\
\hline
\end{tabular}

$\mathrm{CV}$, coefficient of variation among serial cross sections; the reference combination is in italics.

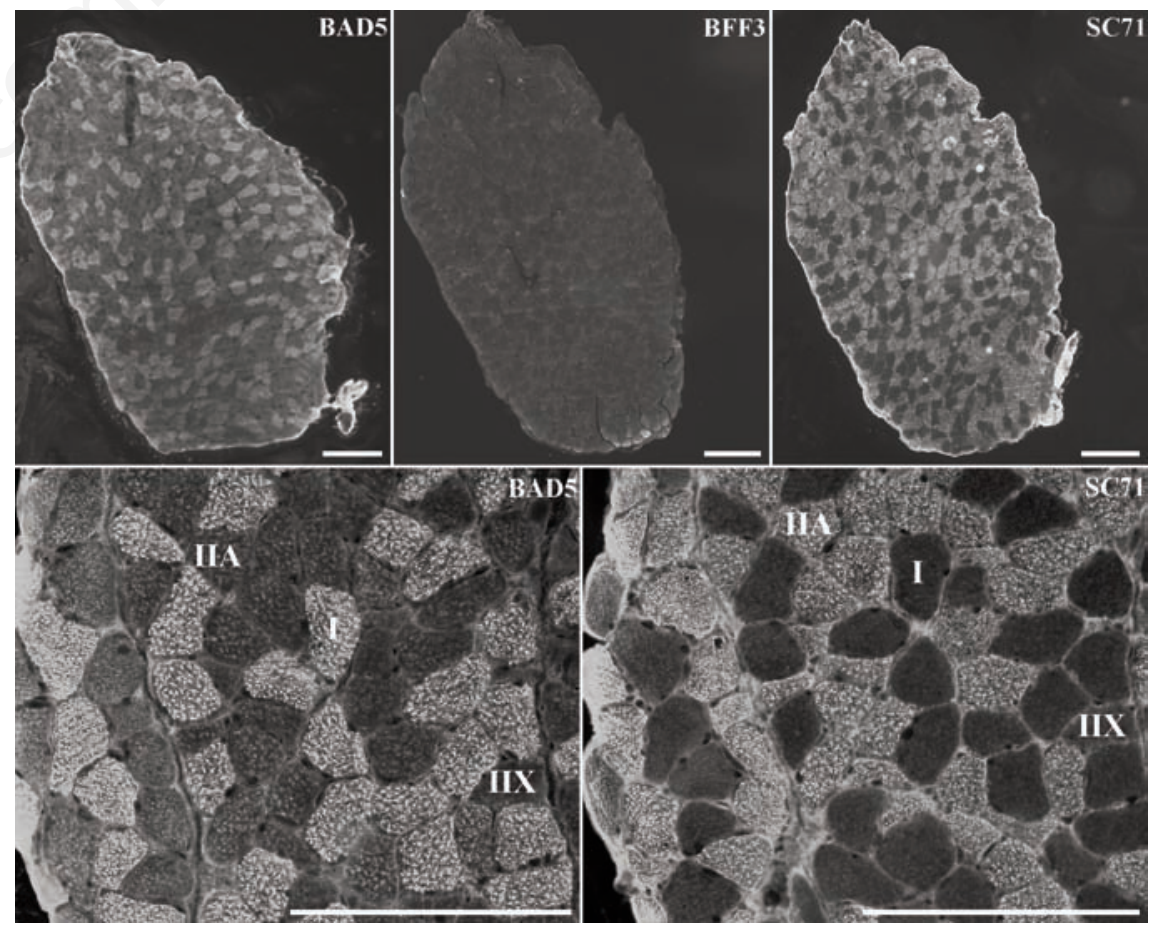

Figure 1. Serial sections of $\mathrm{m}$. Soleus, stained with antibodies specific to MyHC I (BAD5), MyHC IIb (BF-F3) and MyHC IIa (SC71). Scale bar: $200 \mu \mathrm{m}$. 
fibres and had a higher density of fibres than the TA (511 fibres per $\mathrm{mm}^{2}$ on average). In the TA muscle, the IIB fibres had significantly the largest CSA and the IIA the lowest (Table 6). In the SOL, types I and IIX fibres had significantly the largest area compared to the IIA fibres.

\section{Relationships between immunohis- tochemical classification of fibres and electrophoretic separation of MyHCs isoforms}

In order to validate our classification we compared the immunohistochemical data with the proportion of each MyHC determined by electrophoresis for both muscles. We separated the four MyHC isoforms: fast (IIa, IIx, IIb) and slow (I) isoforms (Figure 4). The relative abundances of the MyHC isoforms separated by electrophoresis (Table 5) was compared to the classification of fibres obtained with the immunohistochemical technique for both muscles (Figure 5 A,B). They showed not significant differences. Electrophoresis detected the presence of MyHC IIb in the SOL which was not revealed by immunohistochemistry.

\section{Discussion}

Our objective was to simplify the semi-automatic classification of muscle fibres of Meunier et al., ${ }^{16}$ by using a minimum number of antibodies. As the number of cross sections to compare will be reduced this would simplify the analysis. Moreover the cost of the histological technique would be minimized and it would save analysis time. We show that the use of only two antibodies for each muscle gives the same results than the use of several antibodies. Moreover the proportions of fibres classified with this method have been validated by the electrophoretic separation.

The proportions of the fibre types in the SOL are in agreement with immunohistochemical data from the literature for this muscle in mouse. ${ }^{26}$ Agbulut et al. ${ }^{17}$ showed that the SOL of mouse contained two fast MyHC isoforms (IIa and IIx, 32.2\% and $15.2 \%$ respectively) and MyHC I (53.6\%) as a slow isoform 12 weeks after birth. These authors concluded that in adult mouse, the SOL did not contain IIb MyHC. Moreover, RT-PCR analysis showed that the IIB MyHC transcript was totally absent in the adult SOL. However in our study, the electrophoretic separation of MyHCs allowed the detection of $3 \%$ of IIb isoform in accordance with Bloemberg and Quadrilatero. ${ }^{28}$ The IIb isoform detected by electrophoresis could probably be present only in hybrid IIX-B fibres containing high proportion of MyHC IIx and very low of MyHC IIb. This could explain why they are not detected by using the BF-F3 anti-
Table 5. Muscle fibre type proportions in the $\mathrm{m}$. Soleus and m. Tibialis Anterior of C57BL6J mouse as detected by immunohistochemical and electrophoretic methods.

\begin{tabular}{lcccc} 
& I & IIA & IIX & IIB \\
m. Soleus & & & & \\
Immunohistochemistry & 43 & 46 & 11 & 0 \\
$\quad$ Electrophoresis & 42 & 44 & 12 & 3 \\
m. Tibialis Anterior & & & & \\
Immunohistochemistry & 0 & 6 & 34 & 57 \\
Electrophoresis & 0 & 7 & 29 & 63 \\
\hline
\end{tabular}

The BA-D5/SC71 (m. Soleus) and the BF-F3/SC71 (m. Tibialis Anterior) combinations were used ( $\mathrm{n}=8$ mice). The MyHC isoforms were separated by electrophoresis according to Mizunoya et al. ${ }^{24}$ Immunohistochemical data are expressed as percent of fibre type proportion; electrophoresis data are expressed as percent abundance of each MyHC.

Table 6. Muscle fibre transversal cross-sectional areas in the $\mathrm{m}$. Soleus and $\mathrm{m}$. Tibialis Anterior of C57BL6J mouse.

\begin{tabular}{|c|c|c|c|c|c|}
\hline \multirow[t]{2}{*}{ Muscle } & \multicolumn{5}{|c|}{ Fibre type } \\
\hline & I & IIA & IIA-X & IIX & IIIB \\
\hline \multicolumn{6}{|l|}{$\mathrm{CSA}\left(\mu \mathrm{m}^{2}\right)(\mathrm{SD})$} \\
\hline m. Soleus & $1187(308)^{\mathrm{a}}$ & $992(284)^{\mathrm{a}}$ & $0(0)$ & $1116(390)^{\mathrm{a}}$ & 0 \\
\hline m. Tibialis anterior & $0(0)$ & $768(156)^{\mathrm{c}}$ & $919(190)^{c}$ & $1343(265)^{\mathrm{b}}$ & $2268(436)^{\mathrm{a}}$ \\
\hline
\end{tabular}

CSA, cross-sectional areas; SD, standard deviation; $\mathrm{n}=8$ mice. ${ }^{\mathrm{a}, \mathrm{b}}$ Means with the same letter are not significantly different.

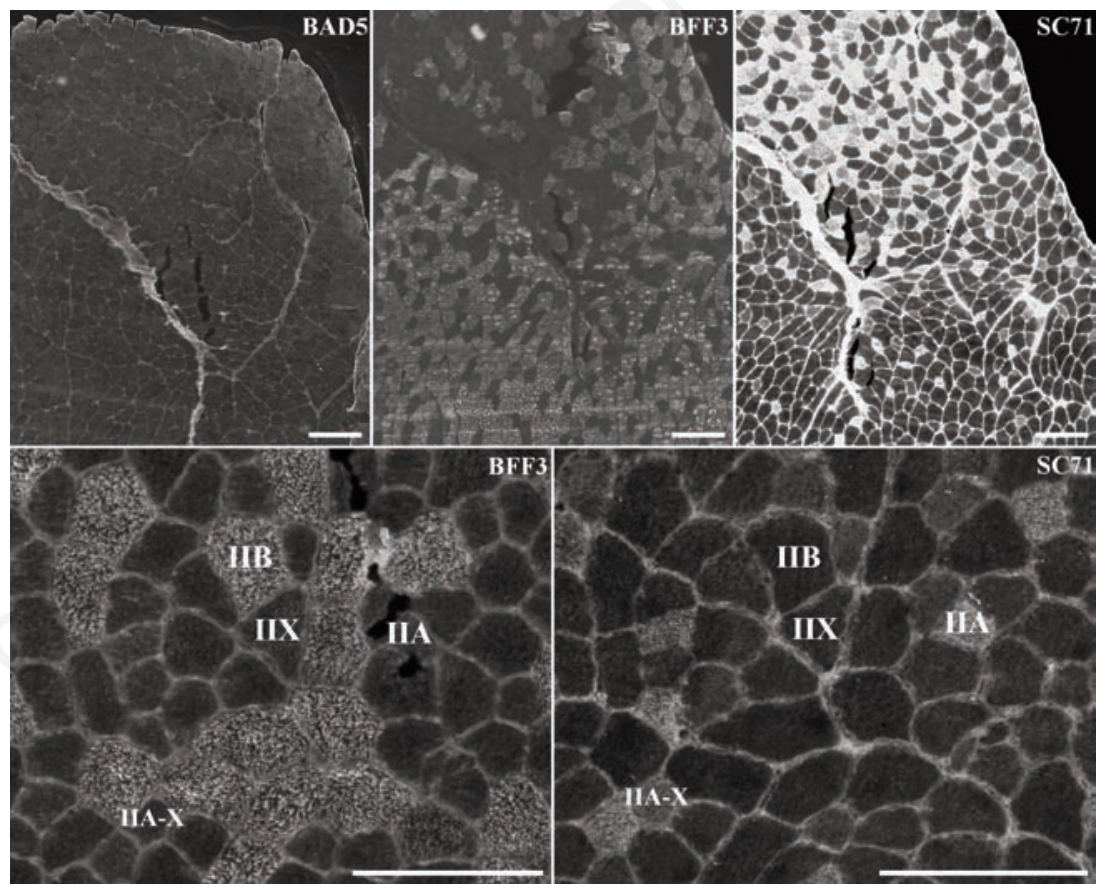

Figure 2. Serial sections of $\mathrm{m}$. Tibialis Anterior stained with antibodies specific to MyHC I (BA-D5), MyHC IIb (BF-F3) and MyHC IIa (SC71). Scale bar: $200 \mu \mathrm{m}$.

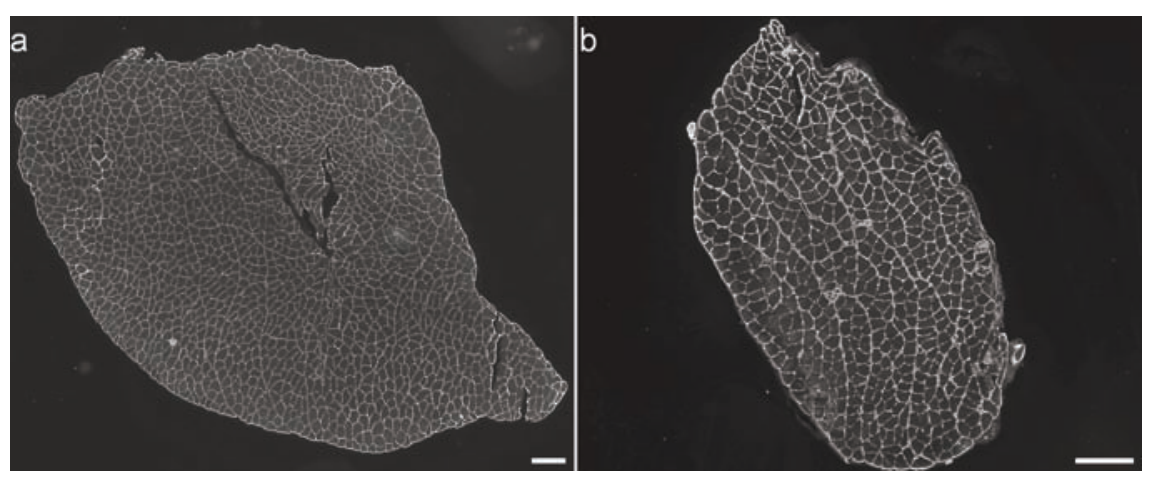

Figure 3. Laminin staining of a) $\mathrm{m}$. Tibialis Anterior, and b) $\mathrm{m}$. Soleus to determine the number of fibres per muscle. Scale bar: $200 \mu \mathrm{m}$. 
body in immunohistochemistry. Some studies have showed that the TA muscle of C57BL6J mouse had predominantly IIB fibres and less abundantly IIA-X, IIX, and IIA fibres. ${ }^{18,25,26}$ Zardini and Parry ${ }^{29}$ also concluded that approximately $1 / 3$ of the fibres in the TA of C57BL6J mouse were IIX type. Our classification is therefore in coherence with these data. One of the advantages of working on the mouse muscles is the possibility to have a section of the whole muscle. This is very interesting to determine the exact total number of fibres per muscle and provides a complete characterization of fibre composition. We show that it is possible for both the SOL and TA at 12 weeks of age. The image analysis allowed to evaluate the cross sectional area of each fibre type. In the SOL we showed that high oxidative fibres (type I) were not the smallest. Their cross sectional areas were similar to that of IIX fibres. In the TA the largest were the IIB fibres. These differences in CSA between the different types of fibres in SOL and TA are in accordance with the data of Bloemberg and Quadrilatero. ${ }^{28}$

All these data give us confidence to validate the use of the two combinations of antibodies proposed for each muscle. Limitations of this classification could be that IIX fibres are classified only on the basis of the absence of labeling. Moreover, in some specific physiological or pathological conditions hybrid IIX-B fibres could be present. Our classification allowed the observation of these hybrid fibres only by differences in labeling intensities with the different antibodies, which may be criticizable. Consequently, for a more accurate analysis we propose to first systematically use the four antibodies BA-D5/SC71/BF-F3/RTD-9 for the two muscles, as illustrated in Figure 6. In SOL the BF-F3 antibody should be used only to validate the absence of IIB fibres. In the TA the BAD5 should be used to validate the absence of type I fibres. If these absences are validated the image-analysis could be based on the combinations of the two antibodies as proposed in this article. If these absences are not validated the image-analysis should be based on the analysis of the three serial sections stained with the three antibodies (BA-D5/SC71/BF-F3). Moreover, for the two muscles the labeling with RTD-9 antibody could be used to validate the IIX or IIX-B fibres.

In conclusion, we propose a simple, fast method of characterization of muscle fibre types and CSA evaluation based on only two anti MyHC antibodies BA-D5/SC71 and BFF3/SC71 in the m. Soleus and the m. Tibialis Anterior respectively. The classification of fibres obtained has been validated by comparison with the literature and with the classification realised by the electrophoretic separation of MyHC in the same samples. The choice of anti MyHC antibodies used for the classifica-

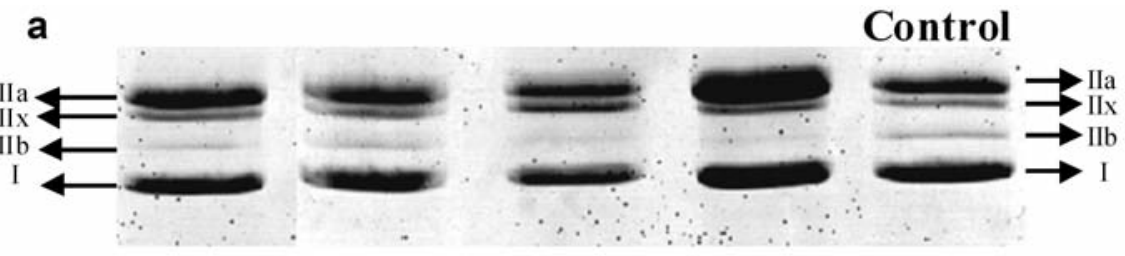

b

Control

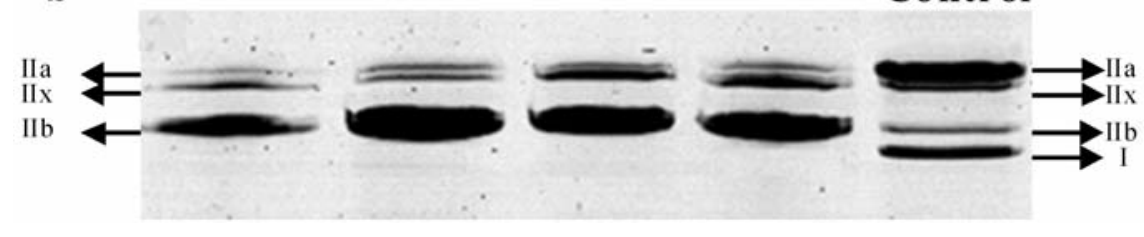

Figure 4. Electrophoretic separation of MyHC in a) m. Soleus, and b) m. Tibialis Anterior. The different wells correspond to different animals. A sample of rat Gastrocnemius with the four types of $\mathrm{MyHC}$ was used as a control.9
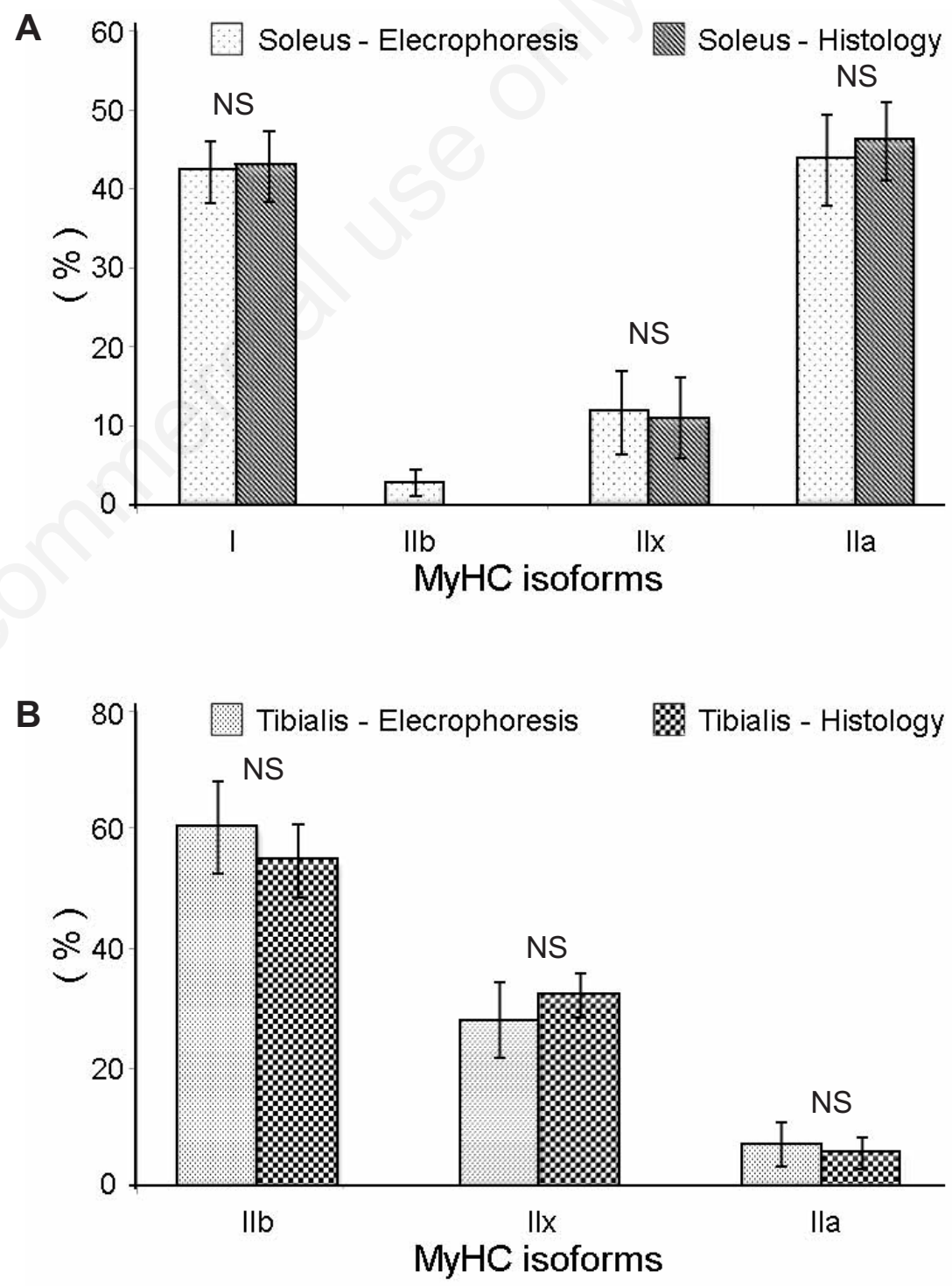

Figure 5. Proportions of the different MyHC isoforms (I, IIa, IIx) detected in A) $\mathrm{m}$. Soleus, and B) m. Tibialis Anterior, with either the histological method or the electrophoretic technique. NS, not significant. 
ANTIBODIES

(SERIAL SECTIONS)

BA-D5, SC71, BF-F3, RTD-9, laminin

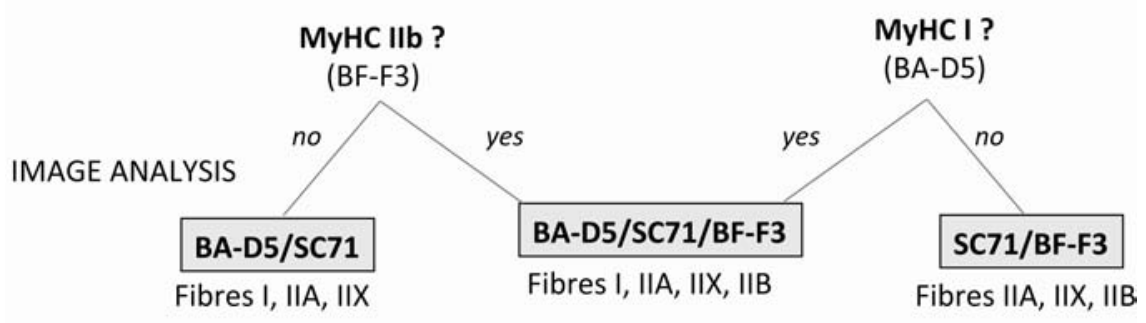

Figure 6. Strategic scheme of choice of the antibodies combinations to be used for each new experiment. It should be adapted for each muscle and each condition. The four antibodies BA-D5, SC71, BF-F3, RTD-9 should be systematically applied with laminin on serial sections. The first step is to choose the combination of antibodies for image analysis. In absence of MyHC IIb, (BF-F3 negative fibres), the BA-D5/SC71 combination should be used to discriminate type I, IIA, IIX and hybrid fibres. In absence of MyHC I (BA-D5 negative fibres), the SC71/BF-F3 combination should be used to discriminate type IIA, IIX, IIB and hybrid fibres. If these absences are not validated by the imageanalysis, the combination BA-D5/SC71/BF-F3 should be used for fibre typing. Labeling with RTD-9 antibody is used only to validate the presence of MyHC IIx in IIX fibres identified by the absence of any labeling.

tion should be adapted for each muscle and each physiological or pathological condition studied. The advantage in mouse is that this immunohistochemical classification can be applied to the total area for muscles with small section areas. This means having information on the total number of fibres, their cross-sectional areas and their composition in absolute value rather than in proportions as in other species. Consequently this method can be applied for studies in which phenotyping of skeletal muscle is necessary, such as in mouse knock-out models.

\section{References}

1. Schiaffino S, Reggiani C. Fiber types in mammalian skeletal muscles. Physiol Rev 2011;91:1447-531.

2. Wydro RM, Nguyen HT, Gubits RM, NadalGinard B. Characterization of sarcomeric myosin heavy chain genes. J Biol Chem 1983;258:670-8.

3. Schiaffino S. Fibre types in skeletal muscle: a personal account. Acta Physiol 2010; 199:451-63.

4. Barany M. ATPase activity of myosin correlated with speed of muscle shortening. J Gen Physiol 1967;50:197-218.

5. Brooke MH, Kaiser KK. Muscle fiber types: how many and what kind? Arch Neurol 1970;23:369-79.

6. Guth L, Samaha FJ. Qualitative differences between actomyosin ATPase of slow and fast mammalian muscle. Exp Neurol 1969; 25:138-52.

7. Peter JB, Barnard RJ, Edgerton VR, Gillespie
CA, Stempel KE. Metabolic profiles of three fiber types of skeletal muscle in guinea pigs and rabbits. Biochemistry 1972;11:2627-33.

8. Reggiani C, Bottinelli R, Stienen GJM. Sarcomeric myosin isoforms: fine tuning of a molecular motor. News Physiol Sci 2000; 15:26-33.

9. Talmadge RJ, Roy RR. Electrophoretic separation of rat skeletal-muscle myosin heavychain isoforms. J Appl Physiol 1993; 75 (5): 2337-2340.

10. Denardi C, Ausoni S, Moretti P, Gorza L, Velleca M, Buckingham M, et al. Type-2xmyosin heavy-chain is coded by a musclefiber type-specific and developmentally-regulated gene. J Cell Biol 1993;123:823-35.

11. Duris MP, Picard B, Geay Y. Specificity of different anti-myosin heavy chain antibodies in bovine muscle. Meat Sci 2000;55:67-78.

12. Smerdu V, Soukup T. Demonstration of myosin heavy chain isoforms in rat and humans: the specificity of seven available monoclonal antibodies used in immunohistochemical and immunoblotting methods. Eur J Histochem 2008;52:179-90.

13. Lu BD, Allen DL, Leinwand LA, Lyons GE. Spatial and temporal changes in myosin heavy chain gene expression in skeletal muscle development. Dev Biol 1999;216: $312-26$

14. Allen DL, Leinwand LA. Postnatal myosin heavy chain isoform expression in normal mice and mice null for IIb or IId myosin heavy chains. Dev Biol 2001;229:383-95.

15. Karen P, Stevanec M, Smerdu V, Cvetko E, Kubinova L, Erzen I. Software for muscle fibre type classification and analysis. Eur J Histochem 2009:53:87-95.

16. Meunier B, Picard B, Astruc T, Labas R.
Development of image analysis tool for the classification of muscle fibre type using immunohistochemical staining. Histochem Cell Biol 2010;134:307-17.

17. Agbulut 0, Noirez P, Beaumont F, ButlerBrowne G. Myosin heavy chain isoforms in postnatal muscle development of mice. Biol Cell 2003;95:399-406.

18. Hamalainen N, Pette D. The histochemical profiles of fast fiber type-IIb, type-IId, and type-Ila in skeletal-muscles of mouse, rat, and rabbit. J Histochem Cytochem 1993; 41:733-43.

19. Schiaffino S, Gorza L, Sartore S, Saggin L, Ausoni S, Vianello M, et al. Three myosin heavy chain isoforms in type 2 skeletal muscle fibres. J Muscle Res Cell Motil 1989;10:197-205.

20. Duris M-P, Picard B, Geay Y. Specificity of different anti-myosin heavy chain antibodies in bovine muscle. Meat Sci 2000; 55 (1): 67-78.

21. Picard B, Barboiron C, Chadeyron D, Jurie C. Protocol for high-resolution electrophoresis separation of myosin heavy chain isoforms in bovine skeletal muscle. Electrophoresis 2011;32:1804-6.

22. Bradford MM. A rapid and sensitive method for the quantitation of microgram quantities of protein utilizing the principle of proteindye binding. Anal Biochem 1976;72:248-54.

23. Laemmli UK. Cleavage of structural proteins during the assembly of the head of bacteriophage T4. Nature 1970;227:680-5.

24. Mizunoya W, Wakamatsu JI, Tatsumi R, Ikeuchi Y. Protocol for high-resolution separation of rodent myosin heavy chain isoforms in a mini-gel electrophoresis system. Anal Biochem 2008;377:111-3.

25. Sugiura T, Murakami N. Separation of myosin heavy-chain isoforms in rat skeletalmuscles by gradient sodium dodecyl sulfatepolyacrylamide gel-electrophoresis. Biomed Res 1990;11:87-91.

26. Augusto V, Padovani CR, Rocha Campos GE. Skeletal muscle fiber types in C57BI6J mice. Braz J Morphol Sci 2004;27:89-94.

27. Schiaffino S, Sagin L, Viel A, Ausoni S, Sartore S, Gorza L. Muscle fiber types identified by monoclonal antibodies to myosin heavy chains, p. 27-34. In: GPL Benzi, N Siliprandi (eds.), Biochemical aspects of physical exercise. Amsterdam, Elsevier: 1986.

28. Bloemberg D, Quadrilatero J. Rapid determination of myosin heavy chain expression in rat, mouse, and human skeletal muscle using multicolor immunofluorescence analysis. Plos One 2012;7:e35273.

29. Zardini DM, Parry DJ. Identification, distribution, and myosin subunit composition of type-IIx fibers in mouse muscles. Muscle Nerve 1994;17:1308-16. 